\title{
Decarbonylative Cross-Couplings: Nickel Catalyzed Functional Group Interconversion Strategies for the Construction of Complex Organic Molecules
}

\section{Lin Guo and Magnus Rueping*}

Institute of Organic Chemistry, RWTH Aachen University, Landoltweg 1, 52074 Aachen, Germany.

CONSPECTUS: The utilization of carboxylic acid esters as electrophiles in metal-catalyzed cross-coupling reactions is highly demanded as these protocols employ environmentally friendly and readily available ester derivatives as powerful alternatives to the commonly used organohalides. Key challenges that need to be addressed in these transformations are the stability of ester substrates and their high energy barrier for oxidative addition to low-valent metal species. Along with the recent developments of nickel catalysts that allow for oxidative addition with ease, chemists became interested in applying less reactive electrophiles as coupling counterparts in nickel-catalyzed transformations. Hence, our group and others have independently investigated a series of ester group substitutions and functionalizations enabled by nickel catalysis. Such developed methods are of great interest as they allow for the exchange of ester groups which can be used as directing groups in metal-catalyzed $\mathrm{C}-\mathrm{H}$ functionalizations prior their replacement.

In this Account, we summarize our recent efforts toward the development of nickel-catalyzed decarbonylative cross-coupling reactions of carboxylic esters. Achievements accomplished by other groups in this area are also included. So far, a number of new transformations have been 
successfully developed, including decarbonylative alkylations, cyanations, silylations, borylations, aminations, thioetherifications, stannylations, and hydrogenolysis reactions. The above mentioned methods proceed via a nickel-catalyzed decarbonylative pathway and have shown high reactivity, chemoselectivity as well as unique advantages in terms of substrate availability as they employ esters as coupling partners. In combination with the synthesis of natural products and biologically active molecules, the present ester substitution methods should find many applications in the construction of complex frameworks by late-stage modification and functionalization of ester groups.

Although a deep and detailed mechanistic understanding of these types of reactions has not yet been fully achieved, chemists made already great efforts toward establishing the mechanisms at play. Yamamoto initially explored the stoichiometric nickel-mediated decarbonylation process of esters and proposed a reaction mechanism involving $\mathrm{C}($ acyl)-O bond cleavage and $\mathrm{CO}$ extrusion. Moreover, recent DFT studies on the oxidative addition step showed a pronounced ligand effect which results in either $\mathrm{C}(\operatorname{aryl})-\mathrm{C}$ bond or $\mathrm{C}(\operatorname{acyl})-\mathrm{O}$ bond cleavage. While more detailed mechanistic investigations need to be undertaken, the successful development of decarbonylative cross-coupling reactions will serve as a solid basis for further studies.

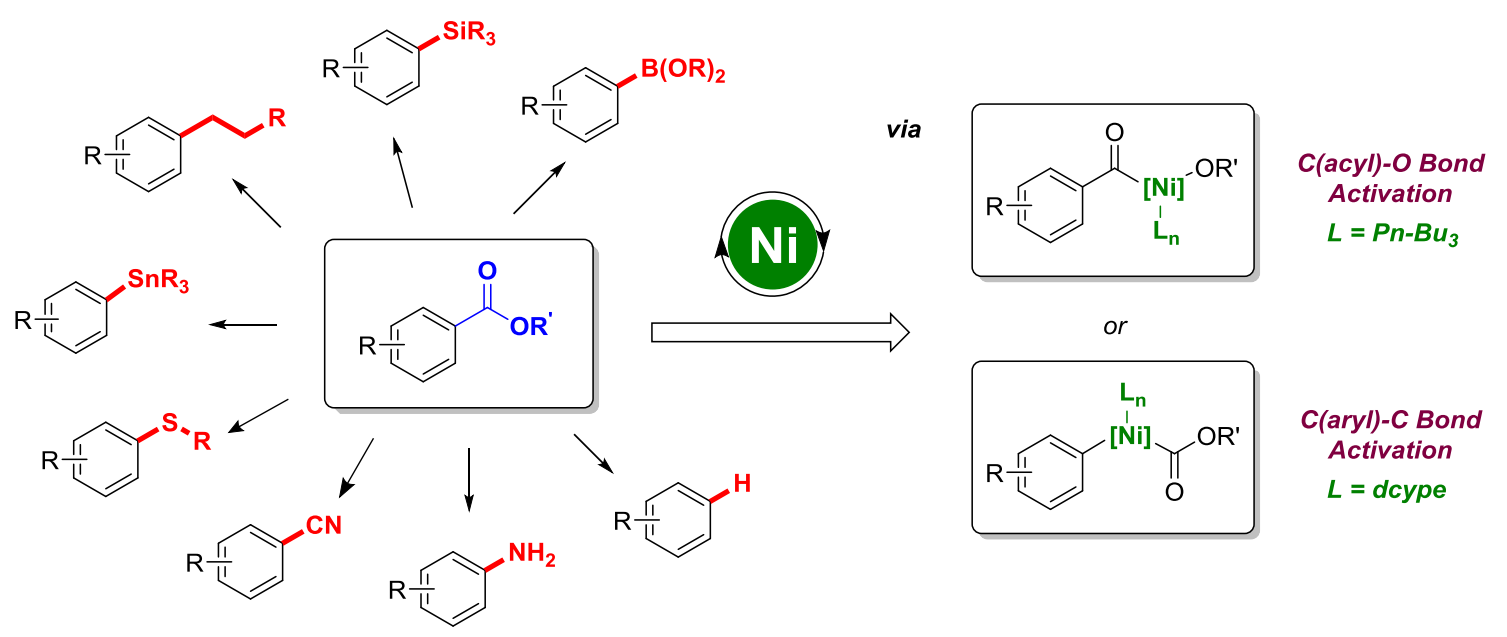




\section{INTRODUCTION}

Cross-coupling reactions have long been recognized as a powerful tool in synthetic applications ranging from natural product synthesis to drug molecule discovery. ${ }^{1}$ Based on the ubiquitous impact of palladium-catalyzed cross-couplings over the past half-century, Heck, Negishi and Suzuki were awarded with the 2010 Nobel Prize in Chemistry. Another group 10 metal in the periodic table is nickel which was not as striking as palladium and was often mistakenly assumed as an inexpensive replacement. ${ }^{2}$ Besides economic considerations nickel has recently become increasingly attractive as chemists recognized its unique characteristics for facile oxidative insertion into carbon-carbon and carbon-heteroatom bonds. This ability enabled the use of electrophiles that are considerably less reactive under palladium catalysis. Owing to this feature the utilization of inert chemical bond-containing electrophiles in nickel catalysis has emerged as a viable and powerful alternative to the classical cross-coupling methods.

Among the existing less reactive electrophiles are esters, a commonly used class of carboxylic acid derivatives which are cheap and abundant in nature. Compared with traditional crosscoupling reactions with aryl halides and sulfonates (Scheme 1a), the utilization of carboxylic acid esters as coupling counterparts in nickel catalysis has received considerable attention not only as it avoids the production of corrosive halide-containing wastes, but since it opens access to the functionalization of a variety of commercially and synthetically available ester-containing molecules. Moreover, the replacement of ester groups is useful in retrosynthetic analysis, as ester groups are considered as suitable directing groups in metal-catalyzed $\mathrm{C}-\mathrm{H}$ functionalization and could be used as such, prior to the replacement. ${ }^{3-6}$ On one hand, the ester group substitution was achieved through nickel-catalyzed cross-coupling via $\mathrm{C}(\operatorname{aryl})-\mathrm{O}$ bond activation (Scheme $1 \mathrm{~b}){ }^{7-9}$ 
The range of nucleophiles that can be coupled with aryl esters and even highly inert aryl ethers has been expanded significantly over the past decade. Another attractive option concerns crosscoupling reactions through a nickel-catalyzed decarbonylative pathway, transformations which will be mainly discussed in this Account (Scheme 1c).

\section{Scheme 1 Overview of Metal-Catalyzed Cross- Coupling Reactions}

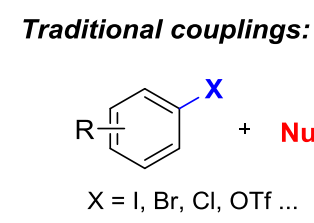

$\mathrm{X}=\mathrm{I}, \mathrm{Br}, \mathrm{Cl}$, OTf ...

Couplings with $\mathrm{C}($ aryl)-O electrophiles:

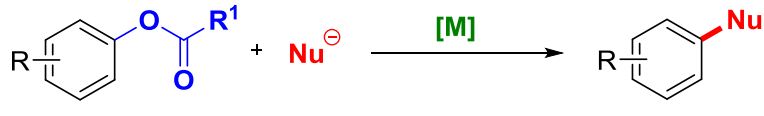

$\mathrm{R}^{1}=t-\mathrm{Bu}, \mathrm{O} t-\mathrm{Bu}, \mathrm{NEt}_{2} \ldots$

Decarbonylative couplings of esters:

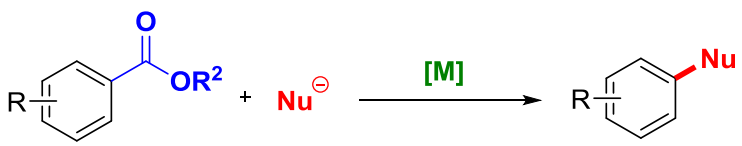

In 1980, Yamamoto and coworkers reported a stoichiometric nickel-mediated decarbonylation process of aryl carboxylates (Scheme 2). ${ }^{10}$ Promoted by a monodentate phosphine nickel complex (e.g. triphenylphosphine), the reaction was proposed to proceed through an oxidative addition mechanism triggered by the nucleophilic attack of the electron-rich nickel complex at the carbonyl group. The following decarbonylation process of carbon monoxide was observed and supported by the isolation and characterization of $\mathrm{Ni}(\mathrm{CO}) \mathrm{L}_{\mathrm{n}}$ complexes. Based on the pioneering work of Yamamoto, carbon-carbon and carbon-heteroatom bond forming reactions proceeding through catalytic decarbonylative cross-coupling of esters and other aroyl derivatives with various nucleophiles have been explored. ${ }^{11-13}$ 


\section{Scheme 2 Initial Study of Nickel-Mediated Decarbonylation of Esters by Yamamoto}

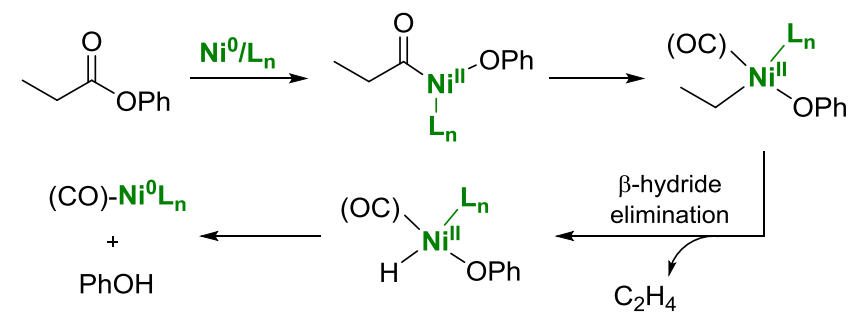

Although many years of research have passed since the initial discovery, a detailed mechanistic understanding of the decarbonylative cross-coupling processes has not been completely achieved. Combining the mechanistic aspects known to date, two mechanisms can be suggested. In the first mechanism proposed (Scheme 3a), the reaction is triggered by oxidative addition of $\mathrm{Ni}(0)$ species $\mathbf{A}$ to the $\mathrm{C}(\mathrm{acyl})-\mathrm{O}$ bond of ester substrates. The resulting acylnickel(II) complex $\mathbf{B}$ subsequently undergoes a decarbonylation step by extrusion of carbon monoxide, generating the arylnickel(II) species C. The transmetalation step is accessed with different nucleophiles and, subsequent reductive elimination releases the corresponding coupling product and regenerates the active $\mathrm{Ni}(0)$ species $\mathbf{A}$. This reaction sequence was nicely illustrated by Shi and coworkers who conducted mechanistic experiments through isolation and characterization of crucial nickel intermediates. ${ }^{14}$ However, disagreement still exist as some reports suggest that transmetalation may occur prior to the $\mathrm{CO}$ extrusion step. ${ }^{15}$ Furthermore, a second mechanistic pathway for $\mathrm{C}(\operatorname{aryl})-\mathrm{C}$ bond cleavage was recently proposed by our group during the investigation of nickelcatalyzed decarbonylative alkylation reaction (see part 2.1 and Scheme $3 b$ ). ${ }^{16}$ DFT studies on the decisive oxidative addition step showed that low-valent nickel species with the support of a bidentate phosphine ligand [e.g. dcype: 1,2-bis(dicyclohexylphosphino)ethane] favorably insert 
into the $\mathrm{C}($ aryl)-C bond, rather than the $\mathrm{C}($ acyl)-O bond. However, more mechanistic studies need to be undertaken to shed light into these intriguing decarbonylative cross-coupling reactions.

\section{Scheme 3 General Proposed Mechanisms for Decarbonylative Cross-Coupling Reactions}

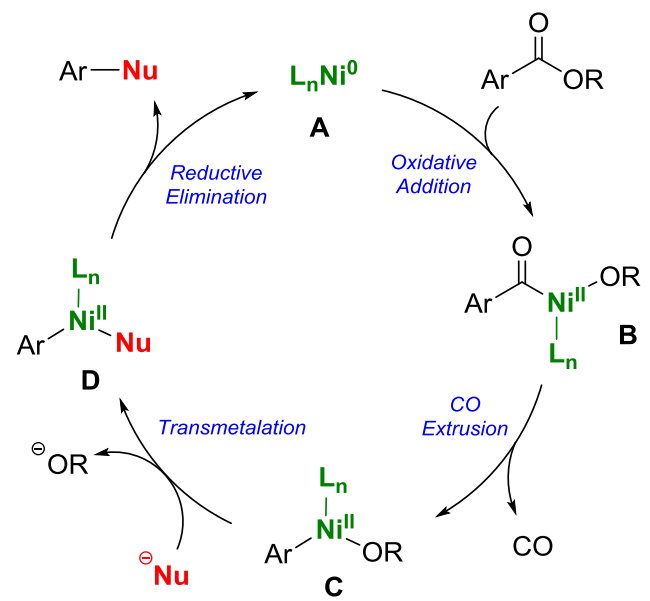

(a) $\mathrm{C}($ acyl)-O bond cleavage

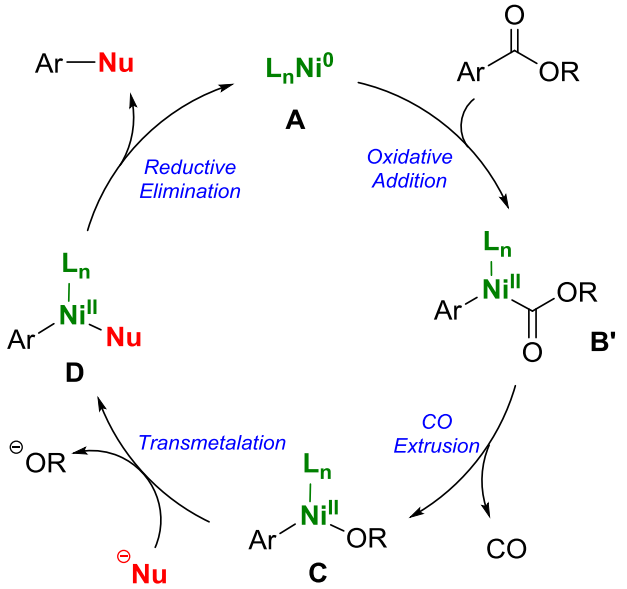

(b) $\mathrm{C}($ aryl)-C bond cleavage

In recent years, we and others have independently developed various new transformations applying this decarbonylative concept. Whereas other related synthetic methods including nickel-catalyzed cross-couplings of redox-active esters via single-electron-transfer (SET) pathway appeared, ${ }^{17-21}$ this Account focuses on the recent progress of nickel-catalyzed decarbonylative cross-coupling reactions via a $\mathrm{Ni}(0) / \mathrm{Ni}(\mathrm{II})$ pathway. The developments in this research area from the discovery of general reaction conditions to the design of new transformations and understanding of reaction mechanisms will serve as a road map for future studies. 


\section{DECARBONYLATIVE CROSS-COUPLING OF ESTERS BY NICKEL CATALYSIS}

\subsection{Decarbonylative $\mathrm{C}_{\mathrm{sp} 2}-\mathrm{C}_{\mathrm{sp} 3}$ Bond Forming Reactions}

Prompted by our initial efforts and achievements in the activation of inert chemical bonds with the aid of nickel catalysis, ${ }^{22-27}$ our group started to focus on the development of new synthetic protocols based on the decarbonylative coupling strategy. We hence questioned whether a decarbonylative $\mathrm{C}_{\mathrm{sp} 2}-\mathrm{C}_{\mathrm{sp} 3}$ cross-coupling reaction could be accessible under nickel catalysis. To achieve this goal, several difficulties had to be considered: i) the spontaneous decomposition of $\mathrm{C}\left(\mathrm{sp}^{3}\right)$-organometallics reagents through proto-demetalation or $\beta$-hydride elimination, ii) ketone formation in the absence of carbon monoxide extrusion, and iii) air and water sensitivity of the $\mathrm{C}\left(\mathrm{sp}^{3}\right)$-organometallic reagents. ${ }^{28}$

In 2017, our group reported the first Ni-catalyzed decarbonylative alkylation reaction of carboxylic ester derivatives with organozinc reagents. ${ }^{29}$ The use of organozinc reagents in the absence of base was expected to be appropriate, meanwhile addressing the challenges towards destructive $\beta$-hydride elimination..$^{30}$ After a series of attempts, a catalytic system consisting of $\mathrm{Ni}(\operatorname{cod})_{2}$ and dcype [1,2-Bis(dicyclohexylphosphino) ethane] as supporting ligand was found to be optimal. Thus, a number of carboxylic esters bearing various aryl or heteroaryl substituents could be coupled with different organozinc compounds in good to excellent yields (Scheme 4). Functional groups, such as methoxy, amine, alkenyl, ketone, and amide, are tolerated under the reaction conditions. Moreover, a secondary alkylzinc reagent, such as cyclohexylzinc bromide, is suitable for this decarbonylative alkylation reaction. 
Scheme 4 Nickel-Catalyzed Decarbonylative $\mathbf{C}_{\text {sp2- }} \mathrm{C}_{\text {sp3 }}$ Bond Formation Using Alkylzinc Reagents as Coupling Nucleophiles
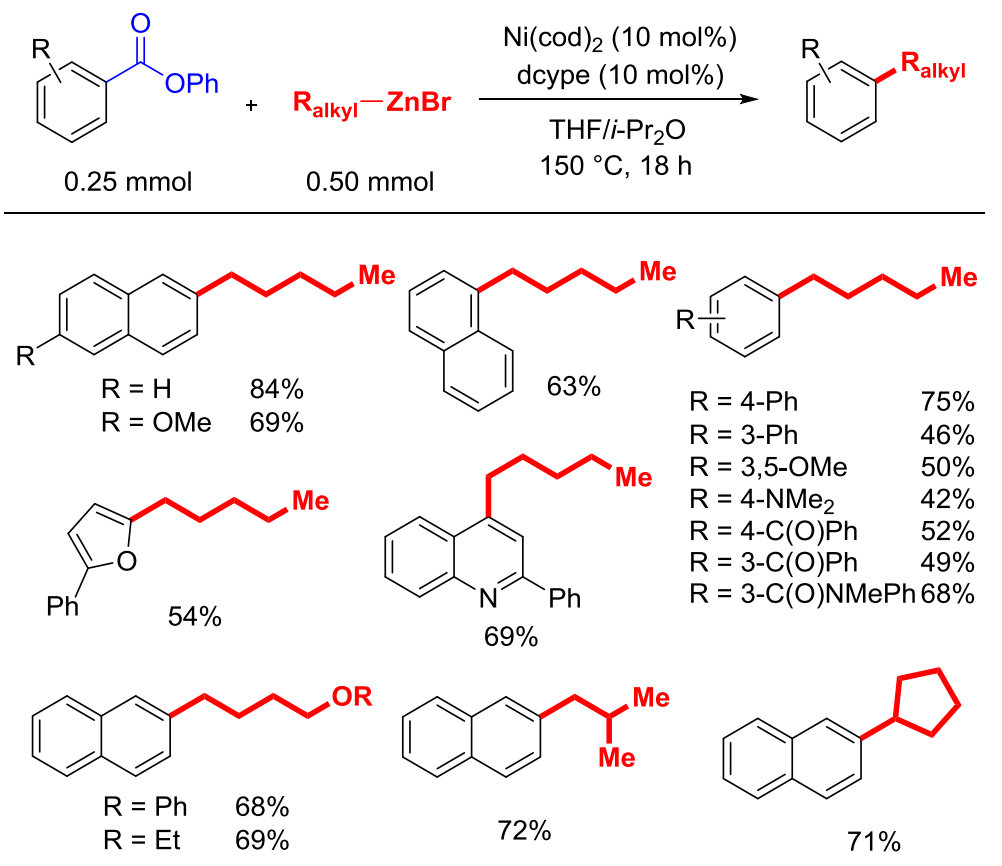

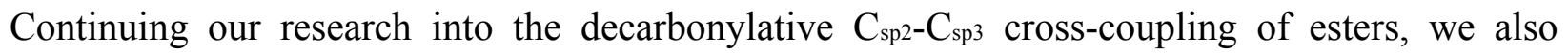
developed a ligand-controlled decarbonylative alkylation protocol by applying $B$-alkyl-9-BBNs as coupling partners. ${ }^{16}$ In this research, $B$-alkyl-9-BBN reagents, which can be generated in situ from the corresponding alkenes via hydroboration, ${ }^{31}$ have shown high efficiency and excellent potential in cross-coupling reactions.

Following the evaluation of different nickel catalysts, ligands, bases, solvents and temperatures, an efficient set of reaction conditions for the coupling of aryl- and heteroaryl-esters with $B$-alkyl9-BBN reagents was identified. Using $10 \mathrm{~mol} \% \mathrm{Ni}(\operatorname{cod})_{2}$ as catalyst, $20 \mathrm{~mol} \%$ dcype as supporting ligand and caesium fluoride or carbonate as base in toluene at $150{ }^{\circ} \mathrm{C}$, the corresponding alkylated products were obtained in good to excellent yields (Scheme 5). 
Scheme 5 Nickel-Catalyzed Decarbonylative $C_{\text {sp2- }} C_{\text {sp3 }}$ Bond Formation Using $B$-alkyl-9-BBNs as Coupling Nucleophiles

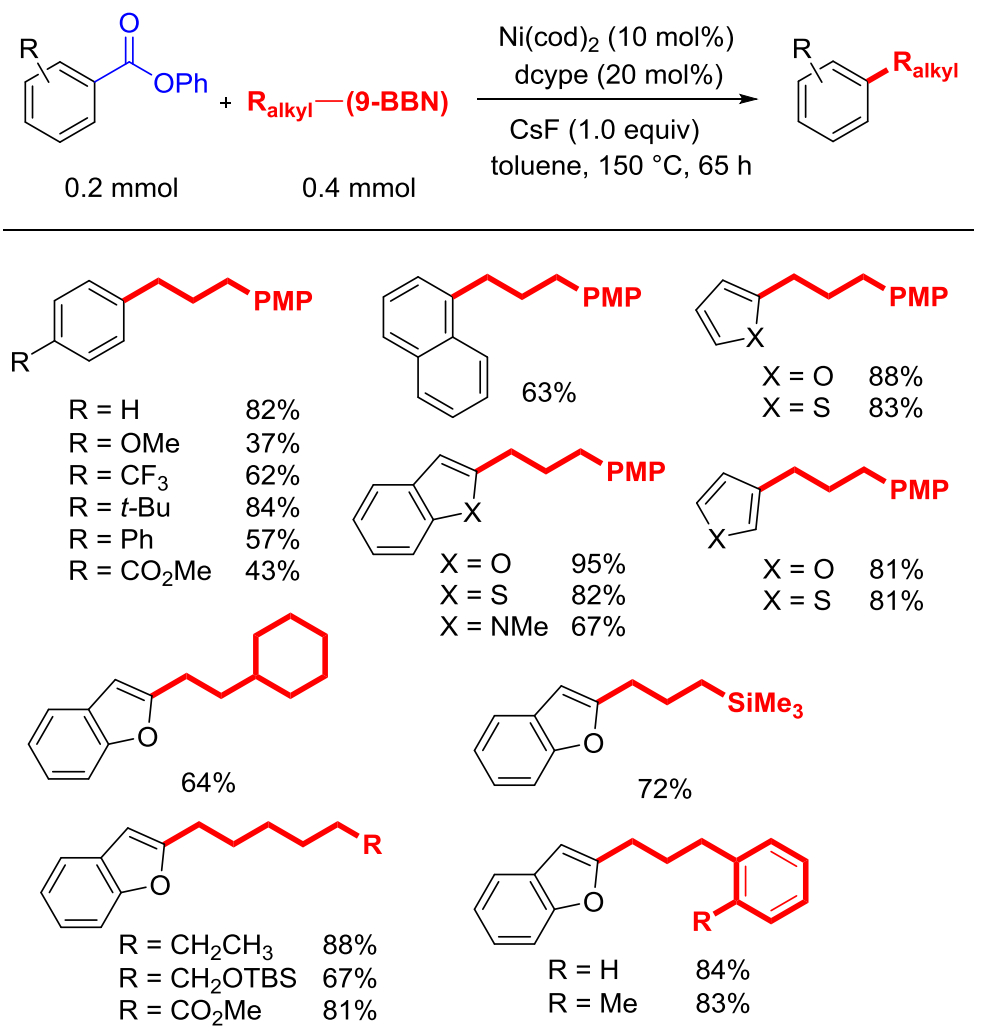

Several features of this cross-coupling reaction are noteworthy. Firstly, the use of bidentate dcype ligand was crucial to accomplish this transformation. The exchange of the bidentate ligand with monodentate phosphine ligands or NHC ligands provided low yields or no product. Secondly, the base was necessary for the formation of an active borate complex which provides a strong driving force in the transmetalation step. ${ }^{32}$ Lastly, this decarbonylative cross-coupling was performed with a wide range of substrates exhibiting functional group tolerance with respect to both esters and alkylboranes. In addition, commercially available triethylborane (BEt 3 ) proved itself an ideal coupling counterpart for this demanding transformation (Scheme 6). 
Scheme 6 Nickel-Catalyzed Decarbonylative $\mathrm{C}_{\text {sp2- }}-\mathrm{C}_{\text {sp3 }}$ Bond Formation Using Triethylborane as Coupling Nucleophiles
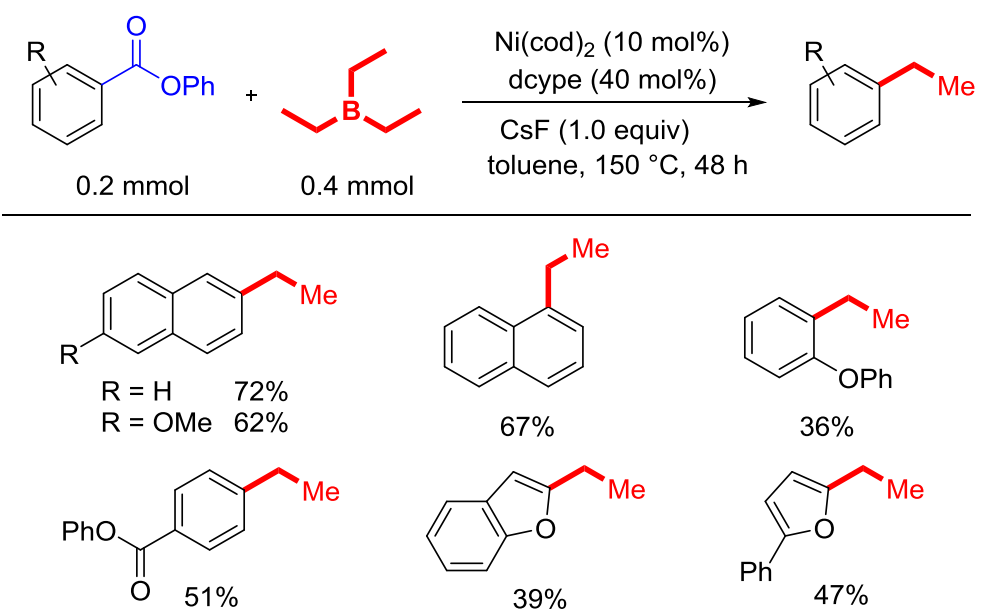

Driven by the observation that a ketone product was mainly isolated if $\mathrm{P} n$ - $\mathrm{Bu}$ 3 ligand (tri- $n$ butylphosphine) was used instead of dcype (Scheme 7), we decided to further explore the ligand effect to the intriguing reaction chemoselectivity. Remarkably, DFT calculations on the decisive oxidative addition step by $\mathrm{Ni}(0)$ species highlighted the different energy profiles if either monodentate or bidentate phosphine ligands were applied (Scheme 8). ${ }^{16,33}$ The calculated results revealed that the ketone product can be favorably generated via $\mathrm{C}(\mathrm{acyl})-\mathrm{O}$ bond activation by nickel complex when mono-dentate phosphine ligands, such as $\mathrm{P} n-\mathrm{Bu}_{3}$ and $\mathrm{PCy}$, were used. However, when the bi-dentate dcype ligand was used, the nickel complex favored the C(aryl)-C bond cleavage in the oxidative addition step (Scheme $8 \mathrm{~b}$ ), leading to the alkylated product via an irreversible decarbonylative process. 
Scheme 7 Ligand-Controlled Ni-Catalyzed Suzuki-Type Cross-Couplings of Esters: C(acyl)-O Bond vs. C(aryl)-C Bond Activation

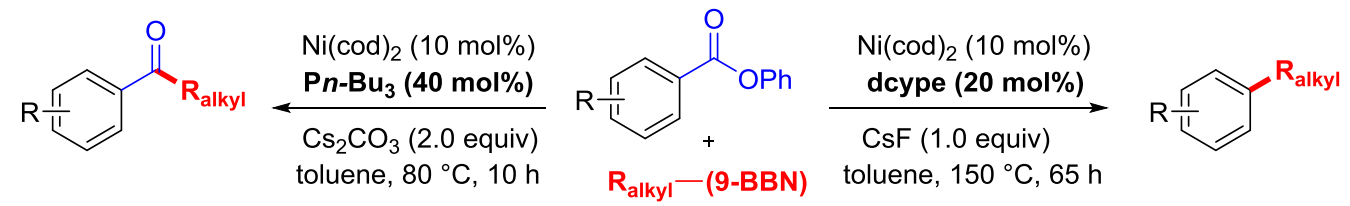

Scheme 8 DFT-Computed Gibbs Free Energies Profile for the Ligand-Controlled NiCatalyzed Oxidative Addition Process by C(acyl)-O Bond and C(aryl)-C Bond Activation.

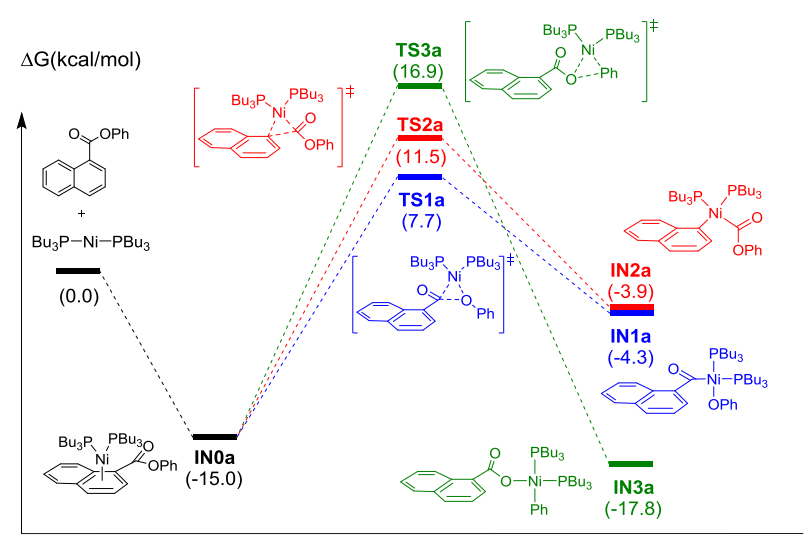

Reaction Coordinate

(a) $\mathrm{Ni}(0) / \mathrm{Pn}-\mathrm{Bu}_{3}$

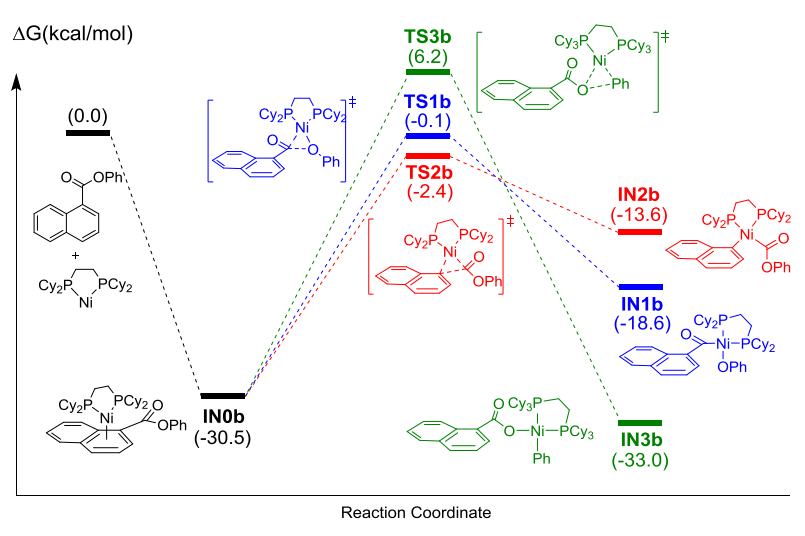

(b) $\mathrm{Ni}(0) /$ dcype

\subsection{Decarbonylative C-CN Bond Forming Reactions}

Having shown for the first time that carboxylic esters can be successfully used for the

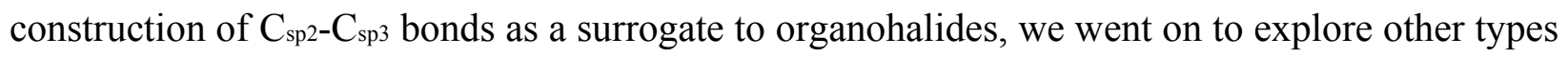
of transformations employing the Ni-catalyzed decarbonylative process. Our attention was drawn to the synthesis of organonitriles, which are important structural motifs in biologically relevant molecules and electronic materials. ${ }^{34}$ Traditional synthetic methods including the Sandmeyer and Rosenmund von Braun reactions may have drawbacks including limited substrate scope and utilization of halides..$^{35,36}$ In contrast, a targeted C-CN bond formation allowing the use of abundant and readily available ester substrates could indeed be a good alternative. With the aim 
of developing the first ester to cyanide functional group interconversion, ${ }^{37}$ we started our investigation and searched for an appropriate nitrile source. After careful evaluation, zinc cyanide as a cheap and harmless cyanating agent provided a successful decarbonylative introduction of the $\mathrm{CN}$ group in the presence of $\mathrm{Ni}(0) / \mathrm{dcype}$ catalytic system. In this case, the scope was broad and different substituents on the aromatic and heteroaromatic moieties were generally accepted (Scheme 9). Interestingly, a series of alkenyl esters containing various electronic properties as well as a $\beta$-substituted alkene derivative also reacted with zinc cyanide to provide alkenyl nitriles in high yields.

\section{Scheme 9 Nickel-Catalyzed Decarbonylative C-CN Bond Formation Using Zinc Cyanide as Coupling Nucleophiles}

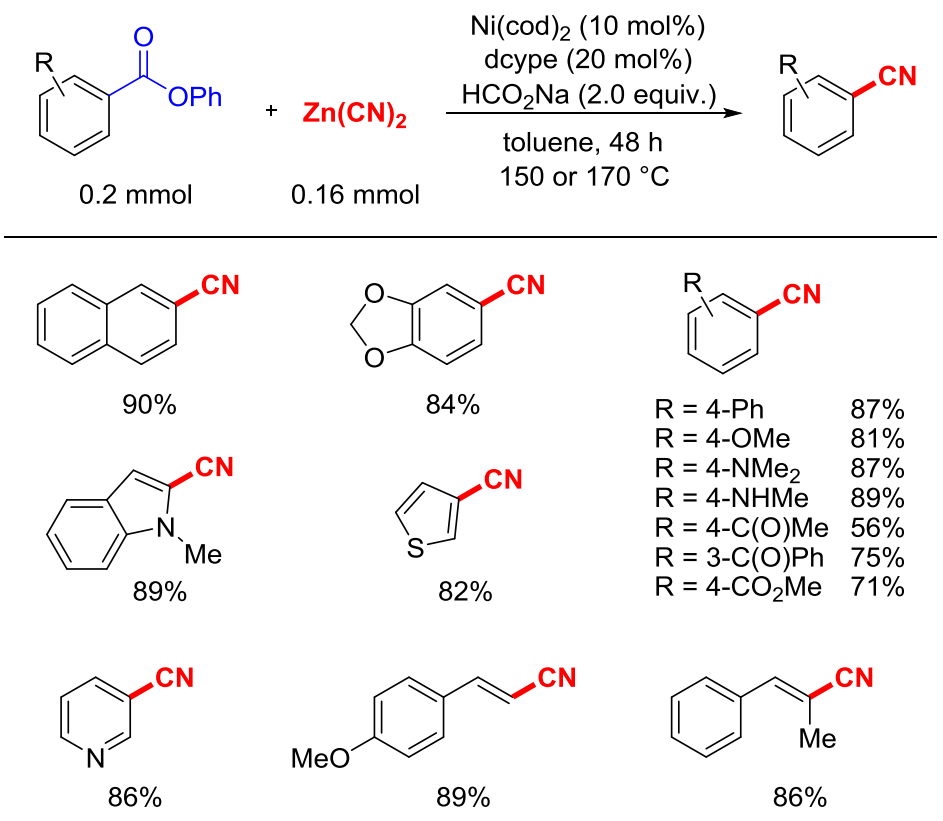

As illustrated for the decarbonylative cyanation reaction of esters, we also aimed for the realization of a deamidative cyanation protocol. In this case, we were delighted to see that the decarbonylative cyanation of twisted amides ${ }^{38}$ proceeded smoothly (Scheme 10A) and the results 
are similar to the simultaneously developed Pd-catalyzed deamidative cyanation protocol by the Szostak group. ${ }^{39}$ Furthermore, an intramolecular recombination fragment coupling of acyl cyanides, which efficiently delivered the corresponding organonitriles in good to moderate yields (Scheme 10B), was developed by our group. ${ }^{37}$ The latter has the advantage that no phenol or imide byproducts are formed; hence, resulting in a cleaner and greener reaction protocol.

\section{Scheme 10 Nickel-Catalyzed Deamidative Cyanation and Intramolecular Cyanation of Acyl Cyanides}

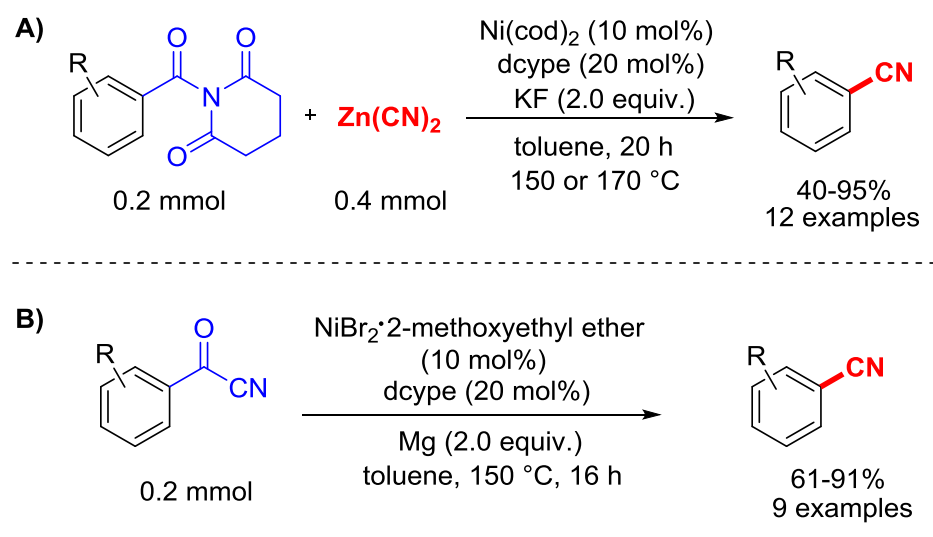

\subsection{Decarbonylative C-Si Bond Forming Reactions}

The role of organosilicon compounds as useful synthetic building blocks has attracted increasing attention from the synthetic community. ${ }^{40-42}$ Traditional methods for the preparation of aryl- and heteroaryl-silanes involve the addition of organolithium or Grignard reagents to chlorosilanes or cyclosiloxanes, ${ }^{43,44}$ transition-metal-catalyzed silyl substitution of halides, ${ }^{45,46}$ and arene C-H silylation. ${ }^{47,48}$ However drawbacks still existed. To this end, chemists are focusing their attention on exploiting novel routes for the synthesis of organosilicon compounds. Thus, we turned our attention to the development of new decarbonylative protocols for carbon-heteroatom bond formation. In this context, a decarbonylative silylation reaction under combined nickel and 
copper catalysis was developed recently by our group (Scheme 11). ${ }^{49}$ After a series of attempts, silylborane compounds were found as efficient silicon nucleophiles owing to their high reactivity and remarkable potential in copper-catalyzed transmetalations. ${ }^{50}$ Even at high temperature, various esters can successfully be coupled with silylborane compounds to give arylsilanes or heteroarylsilanes with excellent functional group tolerance. Heteroaryl esters bearing benzofuran, benzothiophene, indole, quinoline, chromone, furan, pyridine and thiophene motifs, which are often found in bioactive compounds, participated in the reaction, affording the corresponding organosilane products in acceptable yields.

\section{Scheme 11 Nickel-Catalyzed Decarbonylative Silylation Reaction of Esters}

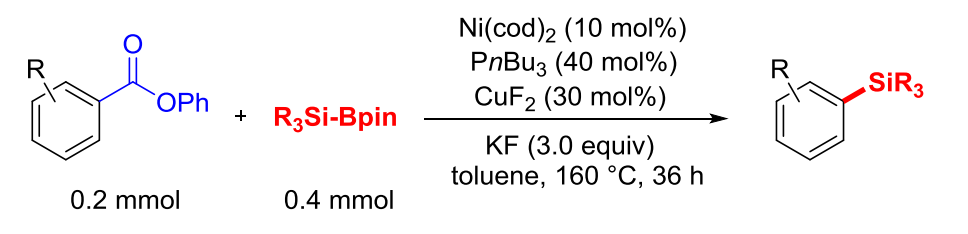

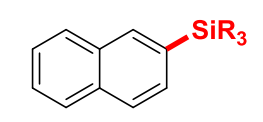

$\mathrm{R}=\mathrm{Et} \quad 87 \%$

$\mathrm{R}=n-\mathrm{Pr} \quad 31 \%$

$\mathrm{R}=\mathrm{Me}_{2} t-\mathrm{Bu} \quad 88 \%$

$\mathrm{R}=\mathrm{Me}_{2} \mathrm{Ph} \quad 95 \%$

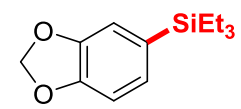

$83 \%$

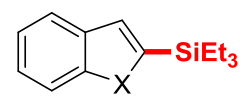

$X=0 \quad 75 \%$

$\mathrm{X}=\mathrm{S} \quad 66 \%$

$\mathrm{X}=\mathrm{NH} \quad 38 \%$

$X=$ NMe $53 \%$

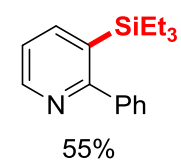

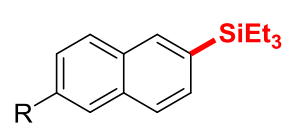

$\mathrm{R}=\mathrm{OMe} \quad 74 \%$

$\mathrm{R}=$ Bpin $\quad 56 \%$

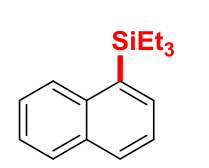

$86 \%$

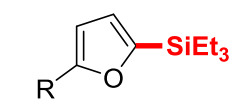

$\mathrm{R}=\mathrm{Ph}$

$\mathrm{R}=\mathrm{CH}=\mathrm{CHPh} 67 \%$

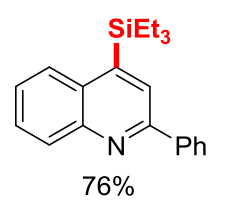

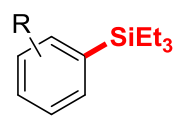

$\mathrm{R}=4-\mathrm{Me} \quad 72 \%$

$\mathrm{R}=4-\mathrm{t}-\mathrm{Bu} \quad 70 \%$

$\mathrm{R}=2-\mathrm{Ph} \quad 64 \%$

$\mathrm{R}=3-\mathrm{Ph} \quad 82 \%$

$\mathrm{R}=4-\mathrm{Ph} \quad 85 \%$

$\mathrm{R}=4-\mathrm{NMe}_{2} \quad 66 \%$

$\mathrm{R}=4-\mathrm{C}(\mathrm{O}) \mathrm{Me} \quad 63 \%$

$\mathrm{R}=4-\mathrm{CO}_{2} \mathrm{Me} \quad 85 \%$

$\mathrm{R}=4-\mathrm{CO}_{2} \mathrm{Ph} \quad 70 \%$

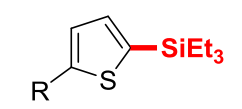

$\mathrm{R}=\mathrm{Ph} \quad 77 \%$

$\mathrm{R}=4-\mathrm{F}-\mathrm{C}_{6} \mathrm{H}_{4} \quad 71 \%$

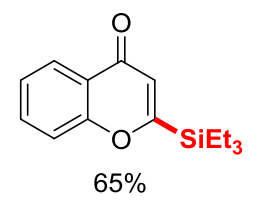


It should be noted that a similar nickel-catalyzed decarbonylative silylation reaction has also been developed by Shi and coworkers (Scheme 13). ${ }^{14}$

Based on these results, a mechanism for the decarbonylative silylation process was proposed, as illustrated in Scheme 12. The initial step of the catalytic cycle involves oxidative addition to $\operatorname{Ni}(0)$ species $\mathbf{A}$ with the support of monodentate phosphine ligand via $\mathrm{C}($ acyl)-O bond insertion. The resulting acylnickel(II) complex $\mathbf{B}$ undergoes a transmetalation route to complex $\mathbf{D}$ facilitated by the in situ generated copper silane complex C. Subsequent decarbonylation and reductive elimination releases the targeted aryl silane while regenerating the $\mathrm{Ni}(0)$ species.

\section{Scheme 12 Proposed Mechanisms for the Ni-} Catalyzed Decarbonylative Silylation Reaction

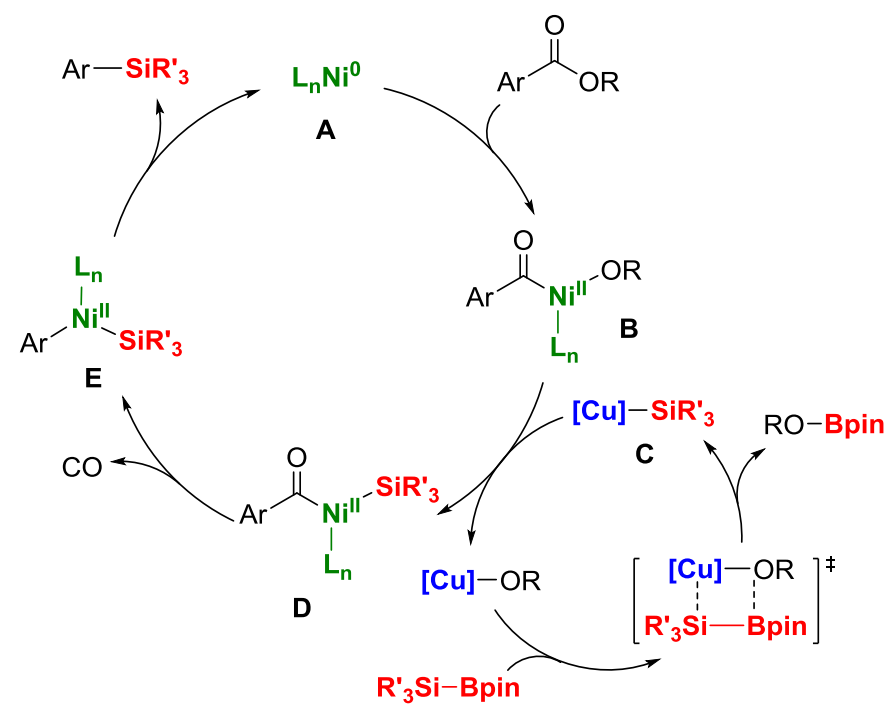




\subsection{Decarbonylative C-B Bond Forming Reactions}

As a continuation of our investigations in this research area, we have next explored the decarbonylative borylation reaction of esters by nickel catalysis. Similar to organosilanes, organoboron compounds are generally recognized as valuable building blocks in natural products synthesis as well as important intermediates in the area of medicinal chemistry, material science and molecular self-assembly. ${ }^{51-53}$ Thus, our group addressed a C-B bond-forming reaction via a decarbonylative process starting from carboxylic esters (Scheme 13). ${ }^{54}$

\section{Scheme 13 Nickel-Catalyzed Decarbonylative Borylation Reaction of Esters}
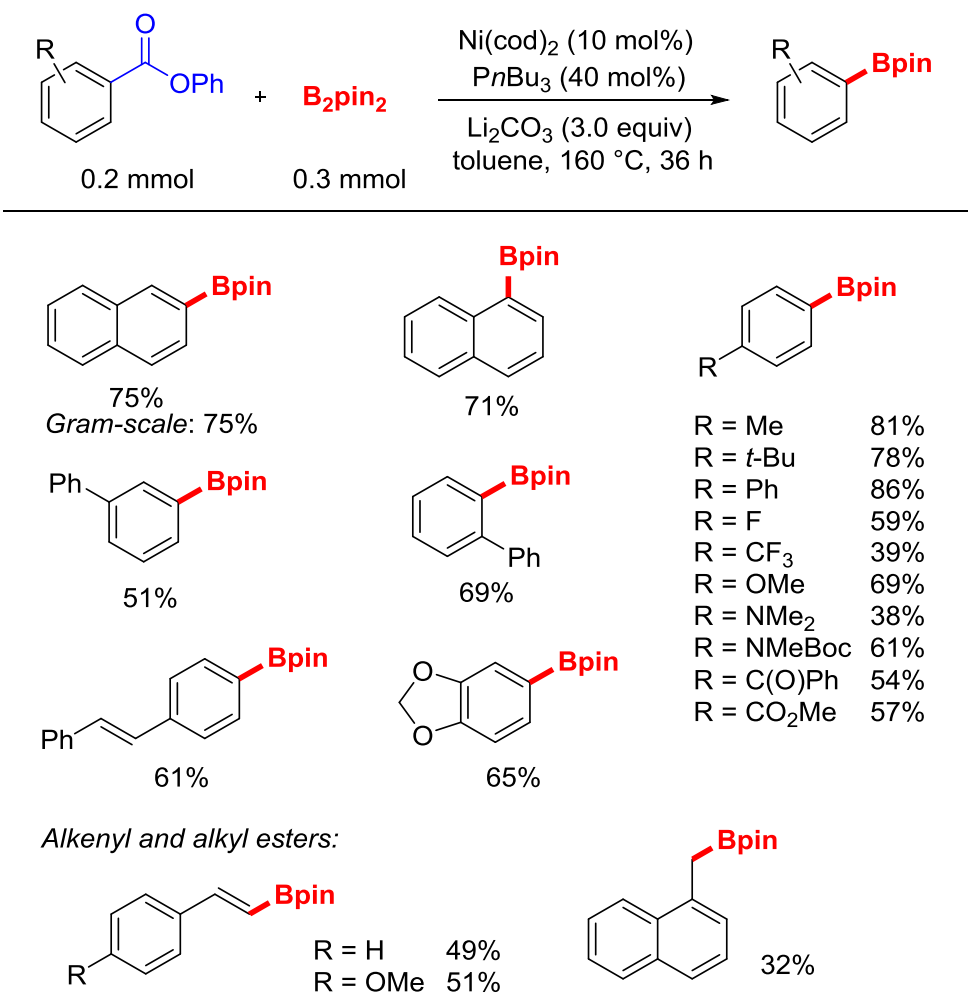

Under the optimized conditions using $10 \mathrm{~mol} \% \mathrm{Ni}(\mathrm{cod})_{2}$ and $40 \mathrm{~mol} \% \mathrm{P} n \mathrm{Bu}_{3}$ various borylated products were efficiently generated. Control experiments revealed that the transformation does 
not proceed without the nickel catalyst or in the absence of the supporting ligand. The optimized reaction conditions tolerated various aryl esters with electron-donating and electron-withdrawing groups as well as alkenyl and alkyl esters. Furthermore, a large scale experiment was conducted with a reduced amount of nickel catalyst and ligand, which emphasizes the applicability of this method. Based on the above mentioned advantages of this protocol, we provide a new possibility for late-stage modification by the replacement of stable ester groups. The resulting boron functionality can be easily functionalized to other valuable products if required. A similar decarbonylative borylation procedure was reported by Shi and coworkers (Scheme 14). ${ }^{14}$

\section{Scheme 14 Nickel-Catalyzed Decarbonylative Borylation Reaction of Esters by Shi}
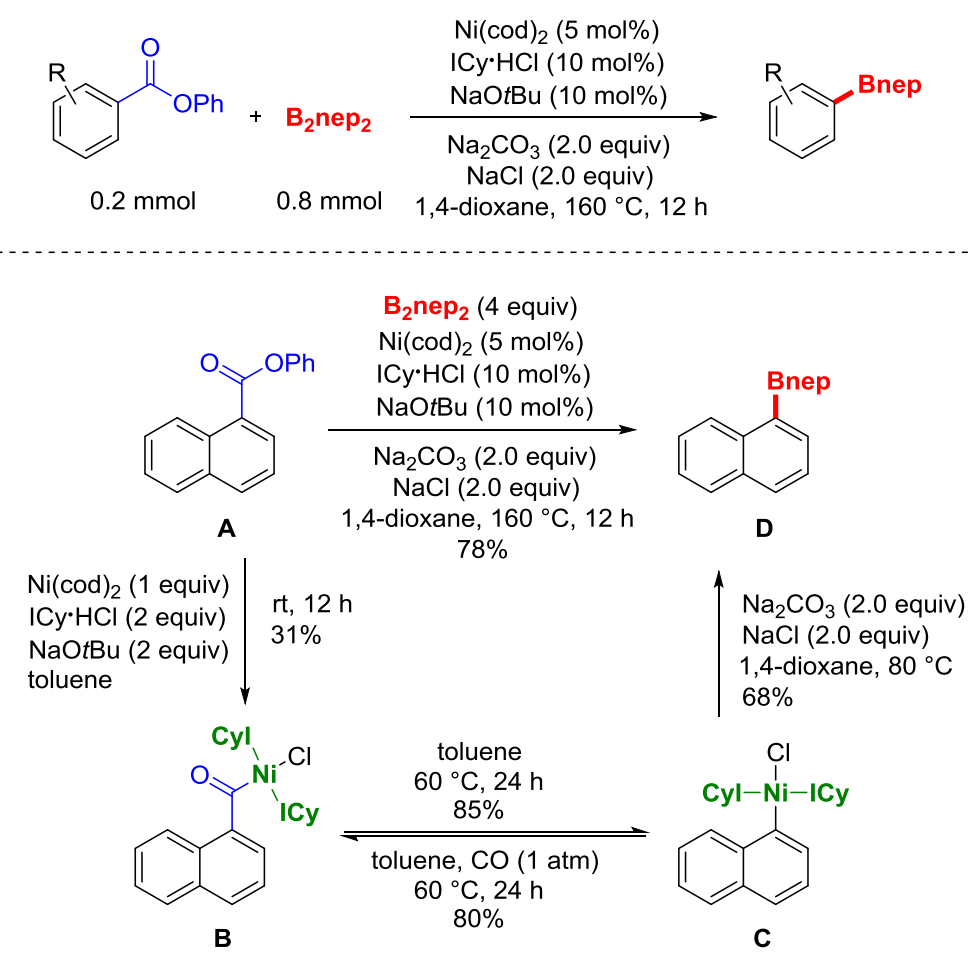

They have developed an efficient $\mathrm{Ni} / \mathrm{ICy} \mathrm{HCl}$ (ICy $\mathrm{HCl}$ : 1,3-dicyclohexyl-imidazolium chloride) catalytic system with $\mathrm{B}_{2}(\mathrm{nep})_{2}$ [Bis(neopentyl glycolato)diboron] as coupling nucleophile which 
is capable of activating the $\mathrm{C}(\mathrm{acyl})-\mathrm{O}$ bonds of esters for decarbonylative borylation to produce various organoboronates. Importantly, mechanistic experiments on this decarbonylative process were carried out and together with two well-characterized nickel-intermediates (B and $\mathbf{C}$, Scheme 14) provided evidences for the ligand-promoted C(acyl)-O bond insertion of nickel.

\subsection{Decarbonylative C-N Bond Forming Reactions}

Having shown that decarbonylative cross-couplings are efficient alternatives to the traditional couplings, we went on to study more frequently and widely applied reaction types. Our attention was drawn to the formation of amines. Primary aniline derivatives represent very useful synthetic intermediates in the construction of biologically active molecules and conjugated functional materials. ${ }^{55,56}$ We envisioned that primary anilines could be accessed by a C-N bond forming event, starting from readily available esters. Such a method would constitute an important alternative to the classical reduction of nitrobenzene derivatives and metal-catalyzed BuchwaldHartwig coupling reactions. ${ }^{57,58}$ As a part of our ongoing studies on ester group replacement, we developed an unprecedented decarbonylative $\mathrm{C}\left(\mathrm{sp}^{2}\right)-\mathrm{N}$ bond formation using nickel catalysis (Scheme 15). ${ }^{59}$ Applying commercially available benzophenone imine as a nitrogen source, ${ }^{60} \mathrm{a}$ series of aryl and heteroaryl esters underwent decarbonylative amination in the presence of $\mathrm{Ni}(0) /$ dcype. Primary anilines were formed after acidic hydrolysis of the imine intermediate. This amination protocol shows high reactivity with broad functional group tolerance, allowing access to a wide range of anilines from readily available esters. Other nitrogen sources, such as morpholine and pyrazole, were also found to be unsuitable for this protocol. 


\section{Scheme 15 Nickel-Catalyzed Decarbonylative Amination Reaction of Esters}

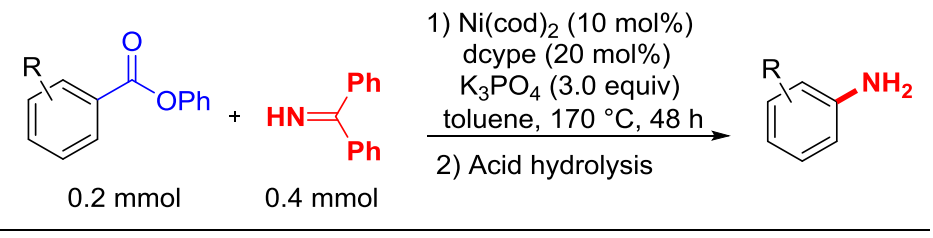

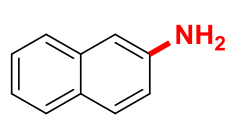

$87 \%$

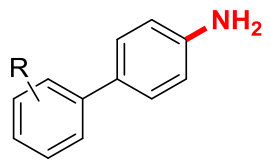

$\mathrm{R}=\mathrm{H} \quad 74 \%$

$\mathrm{R}=4-\mathrm{tBu} \quad 62 \%$

$\mathrm{R}=4-\mathrm{F} \quad 82 \%$

$\mathrm{R}=3,5-\left(\mathrm{CF}_{3}\right)_{2} 61 \%$<smiles>Nc1cccc2ccccc12</smiles>

$63 \%$<smiles>Nc1ccc(-c2ccccc2)s1</smiles>

$60 \%$

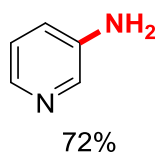

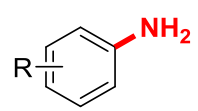

$\mathrm{R}=3-\mathrm{Ph} \quad 73 \%$

$\mathrm{R}=4-\mathrm{OMe} \quad 45 \%$

$\mathrm{R}=4-\mathrm{SMe} \quad 47 \%$

$\mathrm{R}=4-\mathrm{CN} \quad 72 \%$

$\mathrm{R}=4-\mathrm{C}(\mathrm{O}) \mathrm{Me} 56 \%$

$\mathrm{R}=4-\mathrm{CO}_{2} \mathrm{Me} \quad 63 \%$

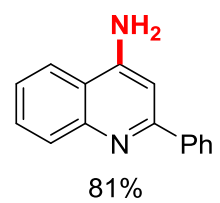

2.6 Decarbonylative C-S Bond Forming Reactions

As the next stage of our studies, we envisioned that a C-S bond coupling of carboxylic esters with thiols might be achieved in this well-developed decarbonylative manner. Although classic transition-metal-catalyzed couplings of thiols with aryl halides are known, these processes still require expensive and reactive eletrophiles as coupling partners. ${ }^{61,62}$ Our group recently reported the first decarbonylative thioetherification reaction of esters using a $\mathrm{Ni}(\mathrm{II}) / \mathrm{dppp}$ system in combination with magnesium as reducing agent. ${ }^{63}$ Remarkably, such a transformation can be performed in a glove-box-free environment, and it represents a crucial breakthrough to achieve C-S bond formation via inert chemical bond cleavage. The substrate scope was very broad, as a 
range of aryl, heteroaryl, and even benzylic esters underwent successfully the decarbonylative CS bond formation with thiols (Scheme 16).

\section{Scheme 16 Nickel-Catalyzed Decarbonylative Thioetherification Reaction of Esters}
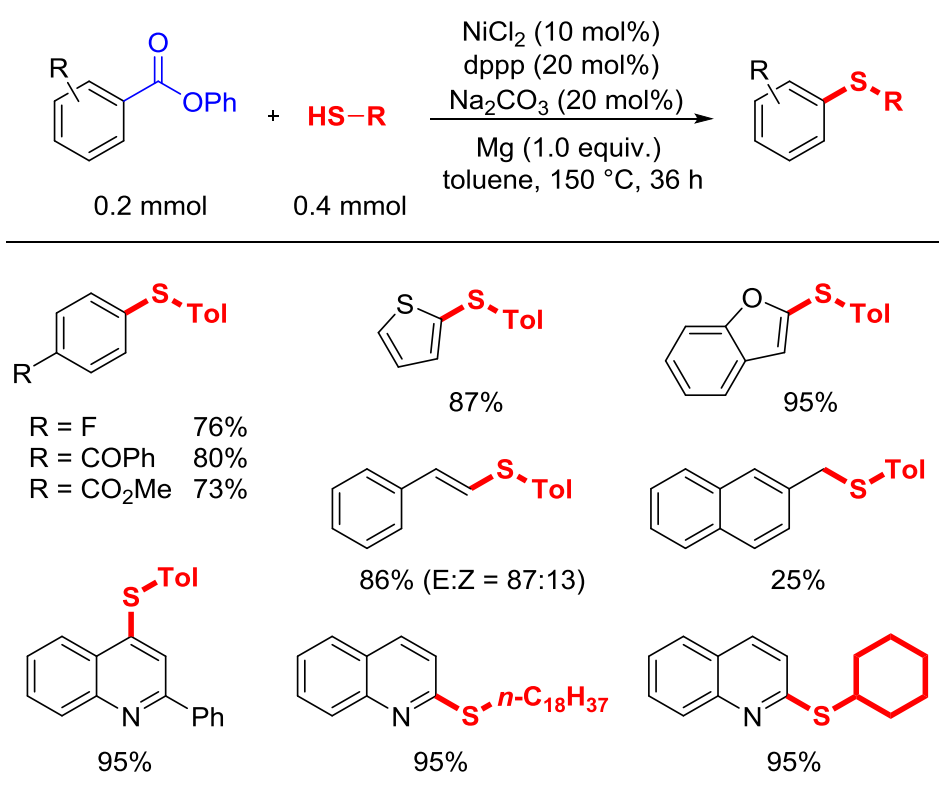

Based on our success in the decarbonylative C-S bond forming reaction by ester group substitution, we expected that the reaction conditions can be further applicable to amides as well as thioesters. To test our hypothesis, various aromatic and heteroaromatic amides were first treated with thiols in the presence of $\mathrm{NiCl}_{2}$ and dppp, giving the corresponding thioethers products in 62-93\% yield (Scheme 17A). An intramolecular decarbonylation and thioetherification was also demonstrated by us as well as the Sanford group (Scheme 17B). ${ }^{64}$ Remarkably, the synthesis of benzothiophene could also be achieved from thiochromen via a Nicatalyzed intramolecular decarbonylative fashion (Scheme 17C). ${ }^{63}$ 


\section{Scheme 17 Nickel-Catalyzed Decarbonylative Thioetherification Reaction of Amides and Thioesters}
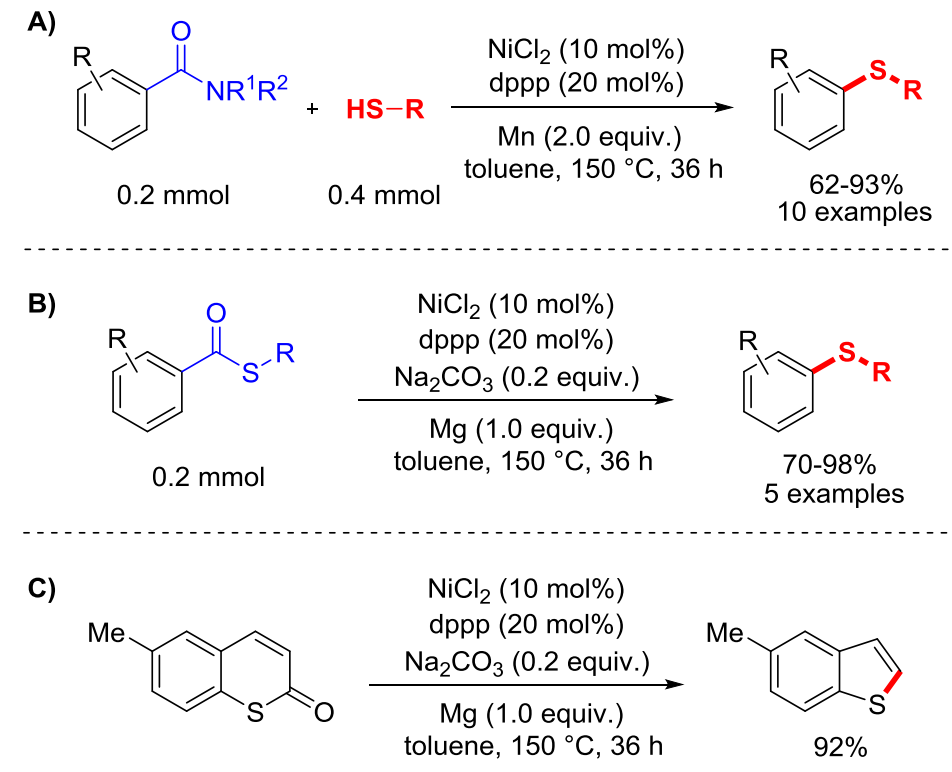

\subsection{Decarbonylative C-Sn Bond Forming Reactions}

Nickel-catalyzed decarbonylative cross-couplings of esters have been well-developed by our group and others. Unlike the wealth of methods on ester substitution of phenyl esters, the means to promote a decarbonylative reaction of methyl esters still remains rather unexplored due to their highly inert nature. Taking into consideration the benefits of applying commercially available and inexpensive methyl esters in cross-coupling reactions, as well as the great significance of organotin compounds in synthetic chemistry, ${ }^{65}$ we recently decided to explore a novel decarbonylative stannylation reaction of methyl esters by nickel catalysis. ${ }^{66}$ The decarbonylative $\mathrm{C}$-Sn bond forming reaction was developed via a Ni-catalyzed C(acyl)-OMe bond activation using $\mathrm{Ni}(\mathrm{cod})_{2} / \mathrm{dppp}$ as catalyst and trimethyl(tributylstannyl)silane as the tin source (Scheme 18A). Notably, a wide range of functional groups such as methyl, fluoro, trifluoromethyl, amide, and methoxy were equally tolerated. Under these conditions, ethyl, 
cyclohexyl, and benzyl esters could also be stannylated, albeit with moderate efficiency. These findings constitute a significant step towards the development of cost-efficient stannylation protocols, allowing for the coupling of more challenging methyl ester substrates. Furthermore, a broad range of phenyl esters was tested under $\mathrm{Ni}(\mathrm{II}) / \mathrm{dppp}$ catalytic conditions and, as expected, these reactions proceeded also in good yields (Scheme 18B).

\section{Scheme 18 Nickel-Catalyzed Decarbonylative Stannylation of Methyl Esters and Phenyl Esters.}
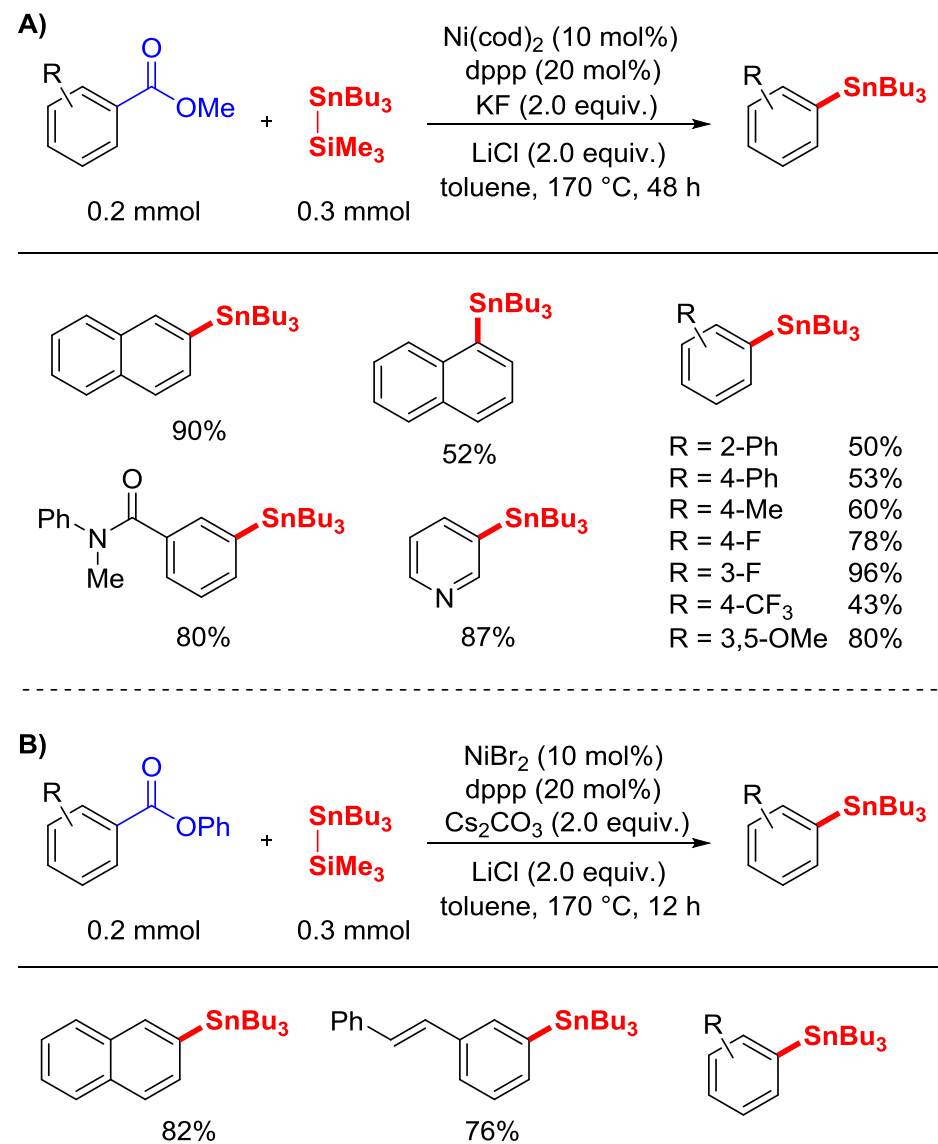<smiles>CCCCc1cccc(C(=O)N([O-])c2ccccc2)c1</smiles>

$70 \%$

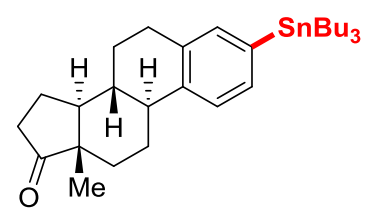

$76 \%$
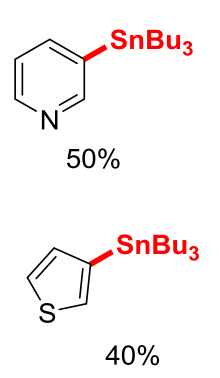

$\begin{array}{ll}\mathrm{R}=\mathrm{H} & 70 \% \\ \mathrm{R}=2-\mathrm{Ph} & 31 \% \\ \mathrm{R}=3-\mathrm{Ph} & 63 \% \\ \mathrm{R}=4-\mathrm{Ph} & 60 \% \\ \mathrm{R}=4-\mathrm{F} & 73 \% \\ \mathrm{R}=3-\mathrm{F} & 70 \% \\ \mathrm{R}=4-\mathrm{CF}_{3} & 60 \% \\ \mathrm{R}=4-\mathrm{CN} & 63 \% \\ \mathrm{R}=4-\mathrm{t}-\mathrm{Bu} & 52 \% \\ \mathrm{R}=3,5-\mathrm{OMe} & 53 \%\end{array}$ 


\subsection{Decarbonylative Hydrogenolysis}

As a next stage of our studies, we decided to move away from nucleophilic substitution of ester groups and instead investigate whether a decarbonylative hydrogenolysis could be achieved. The removal of ester groups could be useful to retrosynthetic analysis, as ester groups are considered as suitable directing groups in metal-catalyzed $\mathrm{C}-\mathrm{H}$ functionalizations. ${ }^{3-6}$ To the best of our knowledge, the removal of ester groups from aromatic and heteroaromatic rings via a decarbonylative pathway was still unknown, ${ }^{67}$ despite the attractiveness of removing functionalities from organic molecules. Inspired by the recent progress in reductive $\mathrm{C}($ aryl $)-\mathrm{O}$ bond cleavage by Martin and Chatani groups, ${ }^{68-70}$ we started to search for a potential reducing agent and finally found that inexpensive, non-toxic and air-stable polymethylhydrosiloxane (PMHS) functions as an efficient reducing agent in the nickel-catalyzed reductive decarbonylation reaction (Scheme 19). ${ }^{71}$ At the same time Lahiri and Maiti developed an elegant deamidative reaction protocol. ${ }^{72}$ In our case $\mathrm{Ni}(\mathrm{OAc}) 4 \mathrm{H}_{2} \mathrm{O}$ was chosen as catalyst. In addition, we found that the bidentate phosphine ligand dcype was the best performing ligand, and the reaction was performed efficiently in the absence of base. As expected, this decarbonylative hydrogenolysis approach is general and the chemoselectivity profile of this process was illustrated by the fact that functional groups, such as methoxy, boronic ester, alkenyl, fluoride, trifluoromethyl, methyl ester, methylthio, ketone, amine and amide moieties were perfectly tolerated under the reaction conditions. Moreover, a great variety of heteroaryl esters bearing benzofuran, indole, quinoline, furan, pyridine and thiophene motifs could also successfully undergo reaction under the optimized hydrogenolysis conditions. 


\section{Scheme 20 Nickel-Catalyzed Decarbonylative Hydrogenolysis of Esters}

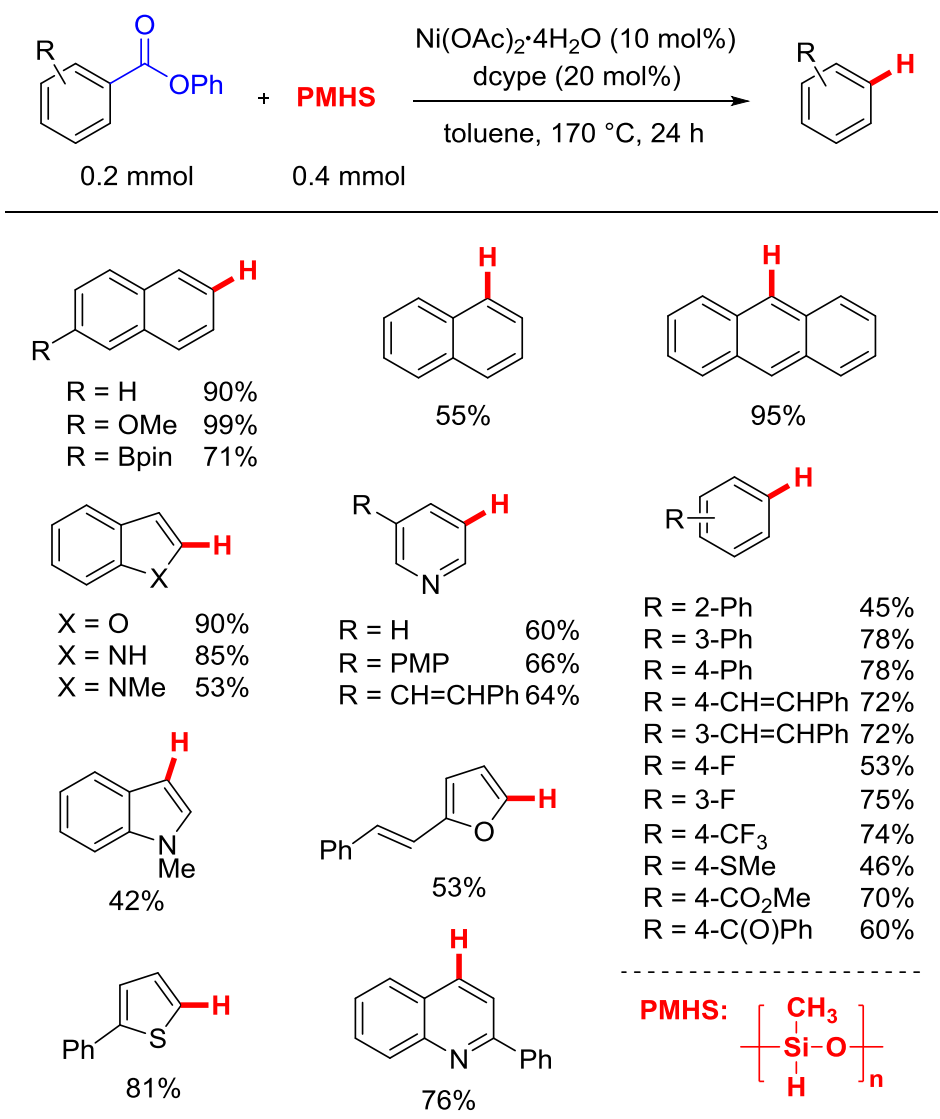

\section{CONCLUSION AND PERSPECTIVE}

Owing to the unique reactivity of nickel that allows for oxidative addition with ease, we and others have recently developed a number of exciting new protocols that have not been achieved with precious-metal catalysts before. In this Account we summarized our recent progress in decarbonylative cross-coupling reactions in which inexpensive and abundant carboxylic acid esters are employed as coupling counterparts (Scheme 20). 


\section{Scheme 20 Overview of Decarbonylative Cross-Coupling Reactions of Ester}

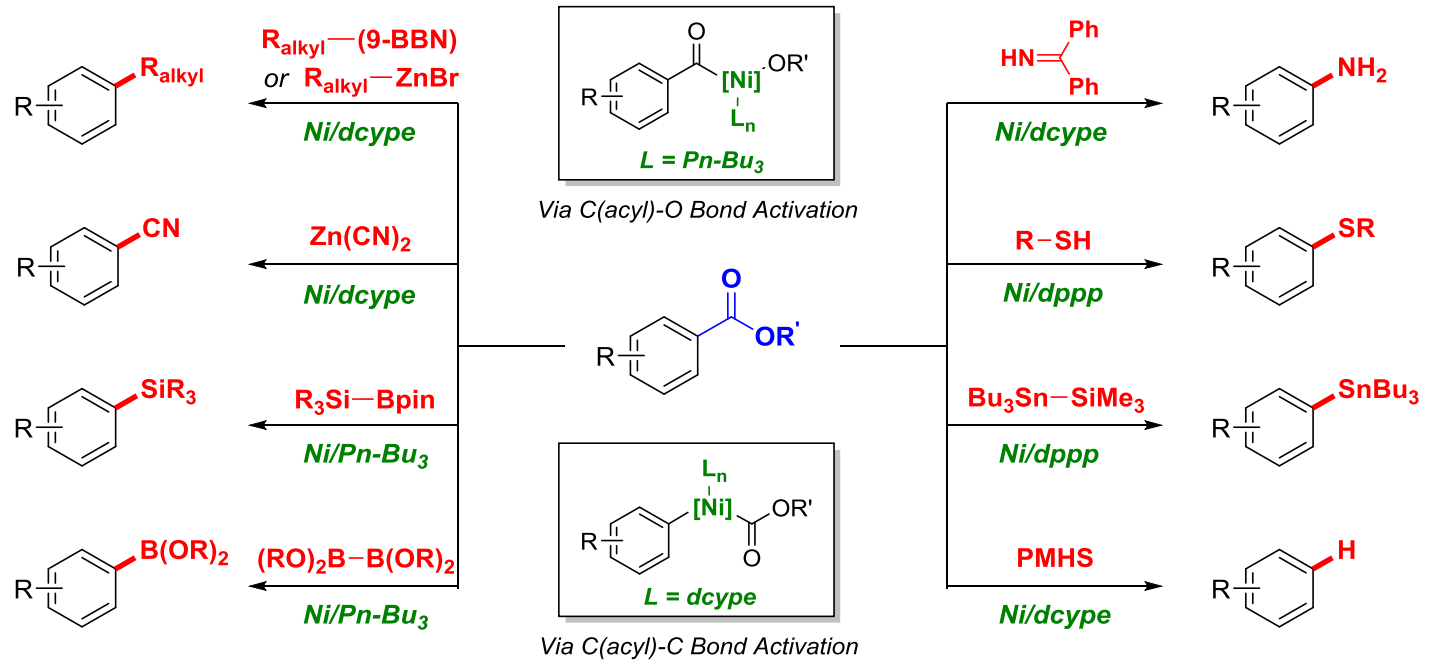

A series of ester group substitutions has been successfully achieved, including decarbonylative alkylation, cyanation, silylation, borylation, amination, thioetherification, stannylation, and hydrogenolysis. Compared with traditional methods using organohalides as coupling electrophiles, these decarbonylative protocols avoid the production of corrosive halidecontaining waste. Furthermore, such developed protocols will be of interest as they allow for the replacement of ester groups, which can be used as directing groups in metal-catalyzed C-H functionalizations prior replacement. Driven by the initial discovery of Yamamoto chemists are still seeking to understand the mechanism of decarbonylative cross-coupling reactions. On one hand the mechanism of $\mathrm{C}(\mathrm{acyl})-\mathrm{O}$ bond insertion by low-valent nickel species with a monodentate phosphine ligand or NHC ligand has been established, and on the other hand, our experimental studies and DFT calculations on oxidative addition step suggest that the $\operatorname{Ni}(0)$ species with a bidentate phosphine ligand insert more likely into $\mathrm{C}($ aryl)-C bond. Nevertheless, all these studies primarily focused on the decisive oxidative addition step due to its high energy barrier, and further efforts towards the mechanistic understanding of the individual 
transformations from both, theoretical and methodological side are still required. Meanwhile, we hope that further new and synthetically useful transformations with respect to the decarbonylative research concept will be developed as the ester functional group interconversion strategy should be of use in retrosynthesis, late stage functionalization and organic synthesis in general.

\section{AUTHOR INFORMATION}

\section{Corresponding Author}

*E-mail: Magnus.Rueping@rwth-aachen.de.

\section{Notes}

The authors declare no competing financial interest.

\section{Biographies}

Lin Guo was born in Shijiazhuang, China. He obtained his B.Sc. from Nankai University in 2011 and M.Sc. from Harbin Institute of Technology in 2013. Currently he is performing his doctoral studies under the supervision of Prof. Magnus Rueping at the RWTH Aachen University, Germany. His research interests focus on catalytic activation of inert chemical bonds and development of novel metal-mediated transformations.

Magnus Rueping studied at the Technical University of Berlin, Trinity College Dublin and ETH Zürich. He obtained his Ph.D. from the ETH in 2002 working with Prof. Dieter Seebach on the synthesis and structural and biological aspects of oligo( hydroxybutanoates) and of $\beta$ - and $\gamma$ - 
peptides. He then moved to Harvard University to work with Professor David A. Evans on enantioselective transition-metal catalysis. In August 2004 he was directly appointed as Associate Professor (C3), the Degussa Endowed Professorship of Synthetic Organic Chemistry, at the University Frankfurt. After four years in Frankfurt he decided to accept a Chair and Full Professorship of Organic Chemistry at RWTH Aachen University. Since 2016 he a member of the KAUST Catalysis Center. His group's research activities are directed toward the development and simplification of synthetic catalytic methodology and technology, and their application in the rapid synthesis of diverse functional natural and unnatural molecules.

\section{ACKNOWLEDGMENT}

L. Guo was supported by the China Scholarship Council.

\section{REFERENCES}

(1) Miyaura, N., Ed. Cross-Coupling Reactions: A Practical Guide; Wiley: Chichester, U.K., 2001.

(2) Tamaru, Y., Ed. Modern Organonickel Chemistry; Wiley-VCH: Weinheim, Germany, 2005.

(3) Trost, B. M.; Imi, K.; Davies, I. W. Elaboration of Conjugated Alkenes Initiated by Insertion into a Vinylic C-H Bond. J. Am. Chem. Soc. 1995, 117, 5371-5372.

(4) Kawamorita, S.; Ohmiya, H.; Hara, K.; Fukuoka, A.; Sawamura, M. Directed Ortho Borylation of Functionalized Arenes Catalyzed by a Silica-Supported Compact Phosphine-Iridium System. J. Am. Chem. Soc. 2009, 131, 5058-5059. 
(5) Park, S. H.; Kim, J. Y.; Chang, S. Rhodium-Catalyzed Selective Olefination of Arene Esters via C-H Bond Activation. Org. Lett. 2011, 13, 2372-2375.

(6) Xiao, B.; Fu, Y.; Xu, J.; Gong, T.-J.; Dai, J.-J.; Yi, J.; Liu, L. Pd(II)-Catalyzed C-H Activation/Aryl-Aryl Coupling of Phenol Esters. J. Am. Chem. Soc. 2010, 132, 468-469.

(7) Cornella, J.; Zarate, C.; Martin, R. Metal-Catalyzed Activation of Ethers via C-O Bond Cleavage: A New Strategy for Molecular Diversity. Chem. Soc. Rev. 2014, 43, 8081-8097.

(8) Su, B.; Cao, Z.-C.; Shi, Z.-J. Exploration of Earth-Abundant Transition Metals (Fe, Co, and Ni) as Catalysts in Unreactive Chemical Bond Activations. Acc. Chem. Res. 2015, 48, 886-896.

(9) Zarate, C.; van Gemmerena, M.; Somerville, R. J.; Martin, R. Phenol Derivatives: Modern Electrophiles in Cross-Coupling Reactions. Adv. Organometal. Chem. 2016, 66, 143-222.

(10) Yamamoto, T.; Ishizu, J.; Kohara, T.; Komiya, S.; Yamamoto, A. Oxidative Addition of Aryl Carboxylates to Nickel(0) Complexes Involving Cleavage of the Acyl-Oxygen Bond. $J$. Am. Chem. Soc. 1980, 102, 3758-3764.

(11) Dzik, W. I.; Lange, P. P.; Gooßen, L. J. Carboxylates as Sources of Carbon Nucleophiles and Electrophiles: Comparison of Decarboxylative and Decarbonylative Pathways. Chem. Sci. 2012, 3, 2671-2678.

(12) Dermenci, A.; Dong, G. Decarbonylative C-C Bond Forming Reactions Mediated by Transition Metals. Sci. China Chem. 2013, 56, 685-701.

(13) Takise, R.; Muto, K.; Yamaguchi, J. Cross-Coupling of Aromatic Esters and Amides. Chem. Soc. Rev. 2017, 46, 5864-5888. 
(14) Pu, X.; Hu, J.; Zhao, Y.; Shi, Z. Nickel-Catalyzed Decarbonylative Borylation and Silylation of Esters. ACS Catal. 2016, 6, 6692-6698.

(15) Muto, K.; Yamaguchi, J.; Musaev, D. G.; Itami, K. Decarbonylative Organoboron CrossCoupling of Esters by Nickel Catalysis. Nat. Commun. 2015, 6, 7508-7515.

(16) Chatupheeraphat, A.; Liao, H.-H.; Srimontree, W.; Guo, L.; Minenkov, Y.; Cavallo, L.; Rueping, M. Ligand-Controlled Chemoselective C(acyl)-O bond vs. C(aryl) $-\mathrm{C}$ bond Activation of Aromatic Esters in Nickel Catalyzed C $\left(\mathrm{sp}^{2}\right)-\mathrm{C}\left(\mathrm{sp}^{3}\right)$ Cross-Couplings. manuscript in revision.

(17) Qin, T.; Cornella, J.; Li, C.; Malins, L. R.; Edwards, J. T.; Kawamura, S.; Maxwell, B. D.; Eastgate, M. D.; Baran, P. S. A General Alkyl-Alkyl Cross-Coupling Enabled by Redox-Active Esters and Alkylzinc Reagents. Science, 2016, 352, 801-805.

(18) Cornella, J.; Edwards, J. T.; Qin, T.; Kawamura, S.; Wang, J.; Pan, C.-M.; Gianatassio, R.; Schmidt, M.; Eastgate, M. D.; Baran, P. S. Practical Ni-Catalyzed Aryl-Alkyl Cross-Coupling of Secondary Redox-Active Esters. J. Am. Chem. Soc. 2016, 138, 2174-2177.

(19) Wang, J.; Qin, T.; Chen, T.-G.; Wimmer, L.; Edwards, J. T.; Cornella, J.; Vokits, B.; Shaw, S. A.; Baran, P. S. Nickel-Catalyzed Cross-Coupling of Redox-Active Esters with Boronic Acids. Angew. Chem. Int. Ed. 2016, 55, 9676-9679.

(20) Edwards, J. T.; Merchant, R. R.; McClymont, K. S.; Knouse, K. W.; Qin, T.; Malins, L. R.; Vokits, B.; Shaw, S. A.; Bao, D.-H.; Wei, F.-L.; Zhou, T.; Eastgate, M. D.; Baran, P. S. Decarboxylative Alkenylation. Nature 2017, 545, 213-218. 
(21) Li, C.; Wang, J.; Barton, L. M.; Yu, S.; Tian, M.; Peters, D. S.; Kumar, M.; Yu, A. W.;

Johnson, K. A.; Chatterjee, A. K.; Yan, M.; Baran, P. S. Decarboxylative Borylation. Science 2017, 356, eaam7355.

(22) Leiendecker, M.; Hsiao, C. C.; Guo, L.; Alandini, N.; Rueping, M. Metal-Catalyzed Dealkoxylative $\mathrm{C}_{\mathrm{aryl}}-\mathrm{C}_{\mathrm{sp} 3}$ Cross-Coupling - Replacement of Aromatic Methoxy Groups of Aryl Ethers by Employing a Functionalized Nucleophile. Angew. Chem. Int. Ed. 2014, 53, 1291212915.

(23) Leiendecker, M.; Chatupheeraphat, A.; Rueping, M. Metal Catalyzed Cross-Coupling of Aryl and Benzyl Methyl Sulfides: Nickel Catalyzed $\mathrm{C}_{\mathrm{aryl}}-\mathrm{C}_{\mathrm{sp} 3}$ and $\mathrm{C}_{\mathrm{sp} 3}-\mathrm{C}_{\mathrm{sp} 3}$ Bond Formations. Org. Chem. Front. 2015, 2, 350-353.

(24) Guo, L.; Leiendecker, M.; Hsiao, C. C.; Baumann, C.; Rueping, M. Nickel Catalyzed Dealkoxylative $\mathrm{C}_{\mathrm{sp} 2}-\mathrm{C}_{\mathrm{sp} 3}$ Cross Coupling Reactions - Stereospecific Synthesis of Allylsilanes from Enol Ethers. Chem. Commun. 2015, 51, 1937-1940.

(25) Liu, X.; Hsiao, C. C.; Kalvet, I.; Leiendecker, M.; Guo, L.; Schoenebeck, F.; Rueping, M. Lewis Acid Assisted Nickel-Catalyzed Cross-Coupling of Aryl Methyl Ethers by C-O BondCleaving Alkylation: Prevention of Undesired $\beta$-Hydride Elimination. Angew. Chem. Int. Ed. 2016, 55, 6093-6098.

(26) Guo, L.; Hsiao, C.-C.; Yue, H.; Liu, X.; Rueping, M. Nickel-Catalyzed $\mathrm{C}_{\mathrm{sp} 2}-\mathrm{C}_{\mathrm{sp} 3}$ CrossCoupling via C-O Bond Activation. ACS Catal. 2016, 6, 4438-4442. 
(27) Guo, L.; Liu, X.; Baumann, C.; Rueping, M. Nickel-Catalyzed Alkoxy-Alkyl Interconversion with Alkylborane Reagents through $\mathrm{C}-\mathrm{O}$ Bond Activation of Aryl and Enol Ethers. Angew. Chem. Int. Ed. 2016, 55, 15415-15419.

(28) Jana, R.; Pathak, T. P.; Sigman, M. S. Advances in Transition Metal (Pd, Ni, Fe)-Catalyzed Cross-Coupling Reactions Using Alkyl-Organometallics as Reaction Partners. Chem. Rev. 2011, $111,1417-1492$.

(29) Liu, X.; Jia, J.; Rueping, M. Nickel-Catalyzed C-O Bond-Cleaving Alkylation of Esters: Direct Replacement of the Ester Moiety by Functionalized Alkyl Chains. ACS Catal. 2017, 7, 4491-4496.

(30) Knochel, P.; Singer, R. D. Preparation and Reactions of Polyfunctional Organozinc Reagents in Organic Synthesis. Chem. Rev. 1993, 93, 2117-2188.

(31) Chemler, S. R.; Trauner, D.; Danishefsky, S. J. The B-Alkyl Suzuki-Miyaura CrossCoupling Reaction: Development, Mechanistic Study, and Applications in Natural Product Synthesis. Angew. Chem. Int. Ed. 2001, 40, 4544-4568;

(32) Matos, K.; Soderquist, J. A.; Alkylboranes in the Suzuki-Miyaura Coupling: Stereochemical and Mechanistic Studies. J. Org. Chem. 1998, 63, 461-470.

(33) Halima, T. B.; Zhang, W.; Yalaoui, I.; Hong, X.; Yang, Y.-F.; Houk, K. N.; Newman, S. G. Palladium-Catalyzed Suzuki-Miyaura Coupling of Aryl Esters. J. Am. Chem. Soc. 2017, 139, $1311-1318$. 
(34) Kleemann, A.; Engel, J.; Kutscher, B.; Reichert, D.; Pharmaceutical Substances: Syntheses, Patents, Applications, 4th ed.; Georg Thieme: Stuttgart, 2001.

(35) Ellis, G. P.; Romney-Alexander, T. M. Cyanation of Aromatic Halides. Chem. Rev. 1987, 87, 779-794.

(36) Anbarasan, P.; Schareina, T.; Beller, M. Recent Developments and Perspectives in Palladium-Catalyzed Cyanation of Aryl Halides: Synthesis of Benzonitriles. Chem. Soc. Rev. 2011, 40, 5049-5067.

(37) Chatupheeraphat, A.; Liao, H.-H.; Lee, S.-C.; Rueping, M. Nickel-Catalyzed C-CN Bond Formation via Decarbonylative Cyanation of Esters, Amides, and Intramolecular Recombination Fragment Coupling of Acyl Cyanides. Org. Lett. 2017, 19, 4255-4258.

(38) Liu, C.; Szostak, M. Twisted Amides: From Obscurity to Broadly Useful Transition- Metal Catalyzed Reactions by N-C Amide Bond Activation. Chem. Eur. J. 2017, 23, 7157-7173.

(39) Shi, S.; Szostak, M. Decarbonylative Cyanation of Amides by Palladium Catalysis. Org. Lett. 2017, 19, 3095-3908.

(40) Denmark, S. E.; Liu, J. H.-C. Silicon-Based Cross-Coupling Reactions in the Total Synthesis of Natural Products. Angew. Chem. Int. Ed. 2010, 49, 2978-2986.

(41) Showell, G. A.; Mills, J. S. Chemistry Challenges in Lead Optimization: Silicon Isosteres in Drug Discovery. Drug Discov. Today 2003, 8, 551-556.

(42) Cheng, C.; Hartwig, J. F. Catalytic Silylation of Unactivated C-H Bonds. Chem. Rev. 2015, $115,8946-8975$. 
(43) Luliński, S.; Serwatowski, J. Bromine as the Ortho-Directing Group in the Aromatic Metalation/Silylation of Substituted Bromobenzenes. J. Org. Chem. 2003, 68, 9384-9388.

(44) Manoso, A. S.; Ahn, C.; Soheili, A.; Handy, C. J.; Correia, R.; Seganish, W. M.; DeShong, P. Improved Synthesis of Aryltrialkoxysilanes via Treatment of Aryl Grignard or Lithium Reagents with Tetraalkyl Orthosilicates. J. Org. Chem. 2004, 69, 8305-8314.

(45) Murata, M.; Suzuki, K.; Watanabe, S.; Masuda, Y. Synthesis of Arylsilanes via Palladium(0)-Catalyzed Silylation of Aryl Halides with Hydrosilane. J. Org. Chem. 1997, 62, 8569-8571.

(46) Denmark, S. E.; Kallemeyn, J. M. Palladium-Catalyzed Silylation of Aryl Bromides Leading to Functionalized Aryldimethylsilanols. Org. Lett. 2003, 5, 3483-3486.

(47) Lu, B.; Falck, J. R. Efficient Iridium-Catalyzed C-H Functionalization/Silylation of Heteroarenes. Angew. Chem. Int. Ed. 2008, 47, 7508-7510.

(48) Cheng, C.; Hartwig, J. F. Rhodium-Catalyzed Intermolecular C-H Silylation of Arenes with High Steric Regiocontrol. Science 2014, 343, 853-857.

(49) Guo, L.; Chatupheeraphat, A.; Rueping, M. Decarbonylative Silylation of Esters by Combined Nickel and Copper Catalysis for the Synthesis of Arylsilanes and Heteroarylsilanes. Angew. Chem. Int. Ed. 2016, 55, 11810-11813.

(50) Oestreich, M.; Hartmann, E.; Mewald, M. Activation of the Si-B Interelement Bond: Mechanism, Catalysis, and Synthesis. Chem. Rev. 2013, 113, 402-441. 
(51) Trippier, P. C.; McGuigan, C. Boronic Acids in Medicinal Chemistry: Anticancer, Antibacterial and Antiviral Applications. Med. Chem. Comm. 2010, 1, 183-198.

(52) Lorbach, A.; Huebner, A.; Wagner, M. Aryl(hydro)boranes: Versatile Building Blocks for Boron-Doped $\pi$-Electron Materials. Dalton Trans. 2012, 41, 6048-6063.

(53) Mastalerz, M. Shape-Persistent Organic Cage Compounds by Dynamic Covalent Bond Formation. Angew. Chem. Int. Ed. 2010, 49, 5042-5053.

(54) Guo, L.; Rueping, M. Functional Group Interconversion: Decarbonylative Borylation of Esters for the Synthesis of Organoboronates. Chem. Eur. J. 2016, 22, 16787-16790.

(55) Ricci, A., Ed. Amino Group Chemistry: From Synthesis to the Life Sciences; Wiley-VCH: Weinheim, 2008.

(56) Rappoport, Z., Ed. The Chemistry of Anilines, Parts 1 and 2; John Wiley \& Sons: New York, 2007.

(57) Shen, Q.; Hartwig, J. F. Palladium-Catalyzed Coupling of Ammonia and Lithium Amide with Aryl Halides. J. Am. Chem. Soc. 2006, 128, 10028-10029.

(58) Green, R. A.; Hartwig, J. F. Nickel-Catalyzed Amination of Aryl Chlorides with Ammonia or Ammonium Salts. Angew. Chem. Int. Ed. 2015, 54, 3768-3772.

(59) Yue, H.; Guo, L.; Liao, H.-H.; Cai, Y.; Zhu, C.; Rueping, M. Catalytic Ester and Amide to Amine Interconversion: Nickel-Catalyzed Decarbonylative Amination of Esters and Amides by C-O and C-C Bond Activation. Angew. Chem. Int. Ed. 2017, 56, 4282-4285. 
(60) Wolfe, J. P.; Ahman, J.; Sadighi, J. P.; Singer, R. A.; Buchwald, S. L. An Ammonia Equivalent for the Palladium-Catalyzed Amination of Aryl Halides and Triflates. Tetrahedron Lett. 1997, 38, 6367-6370.

(61) Beletskaya, I. P.; Ananikov, V. P. Transition-Metal-Catalyzed C-S, C-Se, and C-Te Bond Formation via Cross-Coupling and Atom-Economic Addition Reactions. Chem. Rev. 2011, 111, $1596-1636$.

(62) Ghaderi, A. Advances in Transition-Metal Catalyzed Thioetherification Reactions of Aromatic Compounds. Tetrahedron 2016, 72, 4758-4782.

(63) Lee, S.-C.; Liao, H.-H.; Chatupheeraphat, A.; Rueping, M. A Glove-box-free Synthesis Enalbes the Nickel-Catalysed Thioetherification at Unactivated C(aryl)-C(acyl) Bonds. Chem. Eur. J. 2018 in revision.

(64) Ichiishi, N.; Malapit, C. A.; Woźniak, Ł.; Sanford, M. S. Palladium- and Nickel-Catalyzed Decarbonylative C-S Coupling to Convert Thioesters to Thioethers. Org. Lett., 2018, 20, 44-47.

(65) Cordovilla, C.; Bartolomé, C.; Martínez-Ilarduya, J. M.; Espinet, P. The Stille Reaction, 38 Years Later. ACS Catal. 2015, 5, 3040-3053.

(66) Yue, H.; Zhu, C.; Rueping, M. Catalytic Ester to Stannane Functional Group Interconversion via Decarbonylative Cross-Coupling of Methyl Esters. Org. Lett. 2018, doi: 10.1021/acs.orglett.7b03669.

(67) Andersson, P. G.; Munslow, I. J. Modern Reduction Methods; Wiley: New York, 2008. 
(68) Álvarez-Bercedo, P.; Martin, R. Ni-Catalyzed Reduction of Inert C-O Bonds: A New Strategy for Using Aryl Ethers as Easily Removable Directing Groups. J. Am. Chem. Soc. 2010, $132,17352-17353$.

(69) Cornella, J.; Gómez-Bengoa, E.; Martin, R. Combined Experimental and Theoretical Study on the Reductive Cleavage of Inert C-O Bonds with Silanes: Ruling out a Classical Ni(0)/Ni(II) Catalytic Couple and Evidence for Ni(I) Intermediates. J. Am. Chem. Soc. 2013, 135, 1997-2009.

(70) Tobisu, M.; Yamakawa, K.; Shimasakia, T.; Chatani, N. Nickel-Catalyzed Reductive Cleavage of Aryl-Oxygen Bonds in Alkoxy- and Pivaloxyarenes Using Hydrosilanes as a Mild Reducing Agent. Chem. Commun. 2011, 47, 2946-2948.

(71) Yue, H.; Guo, L.; Lee, S.-C.; Liu, X.; Rueping, M. Selective Reductive Removal of Ester and Amide Groups from Arenes and Heteroarenes through Nickel-Catalyzed $\mathrm{C}-\mathrm{O}$ and $\mathrm{C}-\mathrm{N}$ Bond Activation. Angew. Chem. Int. Ed. 2017, 56, 3972-3976.

(72) Dey, A.; Sasmal, S.; Seth, K.; Lahiri, G. K.; Maiti, D. Nickel-catalyzed Deamidative StepDown Reduction of Amides to Aromatic Hydrocarbons. ACS Catal. 2017, 7, 433-437. 